\title{
Medial Branch Radiofrequency Ablation as an Alternative of Vertebroplasty for Pain Treatment after Vertebral Compression FRACTURES
}

\author{
Laureano Delange-Segura, MD, PhD, Cristina Delange-Izurrategui, Maria Teresa Palomino-Jiménez, MD, PhD, \\ Mariano Fernández-Baena, MD, PhD, and Marisa Rodríguez-Padilla, MD, PhD
}

Vertebral fractures are very frequent among the population affected by osteoporosis, being pain one of the most distressing associated symptoms. The classic treatment of vertebral fractures is, in principle, conservative. However, in the case of persistent pain, vertebral augmentation techniques and radiofrequency ablation (in the event that vertebral augmentation techniques can't be applied) have proved to be useful.

We present the case of a patient who developed severe pain secondary to an acute $\mathrm{T} 2$ and $\mathrm{L} 3$ fracture, in addition to a posterior T6 fracture. It was treated successfully with L3 vertebroplasty and facet joints denervation of T1-T2 and later T5-T6, this being justified by the technical impossibility of performing vertebroplasty on said levels.
The patient was discharged from the hospital with managed pain relief and more than a $50 \%$ reduction in opioids doses. Three months later, she continued experiencing pain relief.

Radiofrequency ablation of the medial branch of facet joints can be useful in the treatment of pain associated with vertebral fractures in patients where vertebral augmentation techniques are contraindicated or as a complementary treatment for persistent pain.

Key words: Vertebral compression fracture, percutaneous vertebroplasty, radiofrequency ablation, medial branch, facet joints, osteoporosis
Vertebral fractures are very frequent among the population affected by osteoporosis, accounting for approximately 7000 fractures per year in the United States (1). Pain is one of the associated symptoms that produce the greatest discomfort for the patient; it can be prolonged and may need an interventionist's treatment (1).

From : Department of Anesthesiology. Pain Clinic. Regional University Hospital of Málaga, Malaga, Spain

Author for correspondence: Laureano Delange Segura, MD, PhD Address: Department of Anesthesiology, Pain Clinic, Regional University Hospital of Málaga, Av Carlos Haya s/n, 29010, Málaga, Spain E-mail: laudelange@hotmail.es

Disclaimer: There was no external funding in the preparation of this manuscript. Conflict of interest: Each author certifies that he or she, or a member of his or her immediate family, has no commercial association (i.e., consultancies, stock ownership, equity interest, patent/licensing arrangements, etc.) that might pose a conflict of interest in connection with the submitted manuscript.
The treatment of vertebral fractures is - in principle - conservative, consisting of analgesics, resting, and bracing (2). When the pain persists, the most frequently used treatments are vertebroplasty and kyphoplasty. Nevertheless, their use is controversial due to the fact that they can lead to complications such as neurological damage, venous embolization, or fracture of underlying vertebral bodies (3). This is the reason why there has been an attempt to develop other techniques for the treatment of pain secondary to vertebral fractures. In this way, the infiltration and posterior radiofrequency ablation of the medial branch (which innervates the facet joints) has demonstrated efficacy in the management of prolonged pain (4).

We present the case of a patient with osteoporosis secondary to severe malnutrition and hospitalacquired pneumonia with a persistent cough, who developed severe pain secondary to an acute T2 


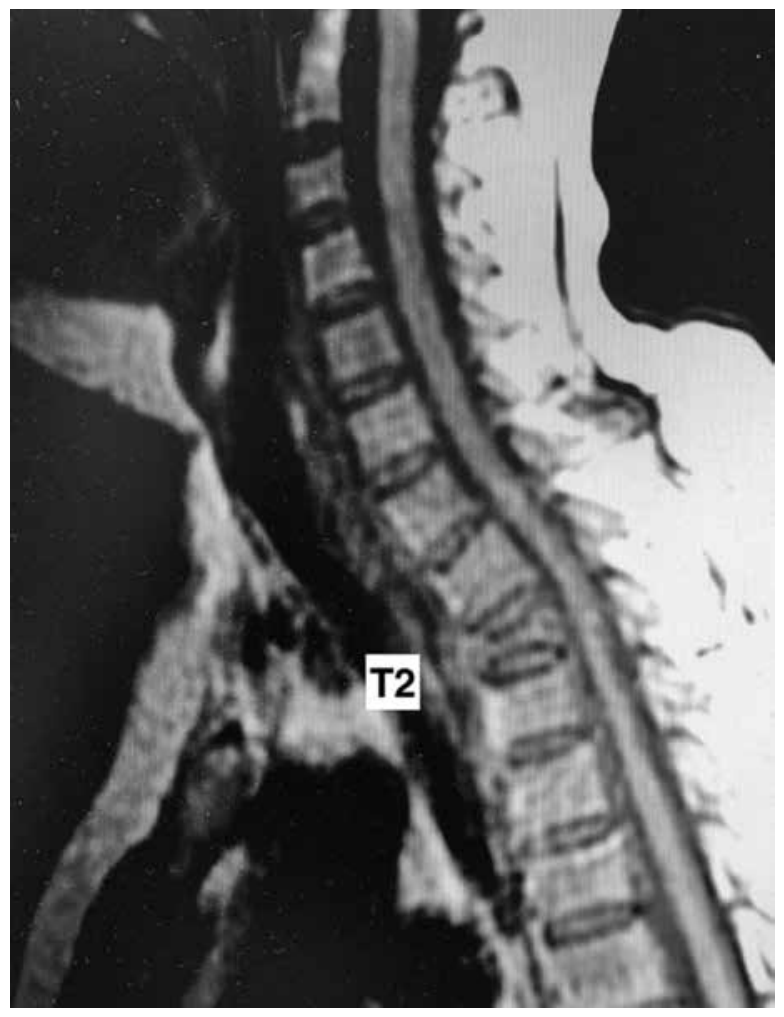

Fig. 1. Compression fracture at T2.

and L3 fracture and, afterward, a T6 fracture. This was treated successfully with L3 vertebroplasty and denervation of the T1-T2 and T5-T6 facet joints. Vertebroplasty could not be performed on said levels due to technical impossibility and failed pain management with high doses of opioids.

\section{CASE DESCRIPTION}

A 48-year-old woman with a history of thrombotic thrombocytopenic purpura, hypothyroidism, gastric medullary carcinoma (in remission) treated with surgery, chemotherapy and radiotherapy, common variable immunodeficiency secondary to radiotherapy, malabsorption with severe malnutrition, short bowel syndrome, and severe osteoporosis with a previous T11 fracture was admitted to hospital for acute cholangitis, where she developed hospital-acquired pneumonia with a persistent cough. Both were managed with antibiotic treatment. Consequently, the

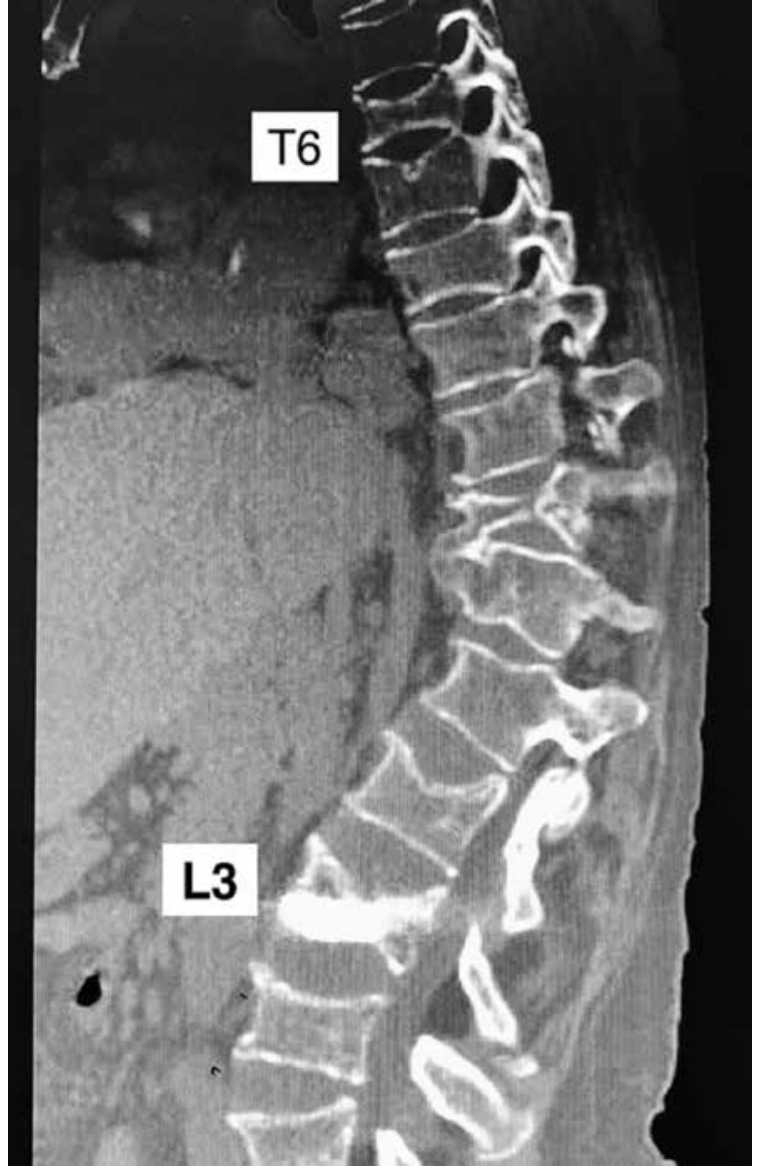

Fig. 2. Compression fracture at L3.

patient started to experience intense pain (Visual Analog Scale [VAS] 8) in the upper back and lumbar region, which became worse during coughing episodes (VAS 10). High doses of opioid treatment (Morphine Equivalent Dose [MED] of $230 \mathrm{mg}$ per day) were administered with poor results.

Magnetic resonance imaging (MRI) and computed tomography (CT) scan revealed compression fractures of her L3 and T2 vertebral bodies (Figs. 1,2). L3 vertebroplasty was performed after consultation with the interventional radiology department. However, T2 vertebroplasty was discarded/rejected because of anatomical structures interposition. Her pain persisted in spite of the opioid treatment; thereby, she was referred to the Pain Unit for possible interventional treatment. After an evaluation, we decided to perform 


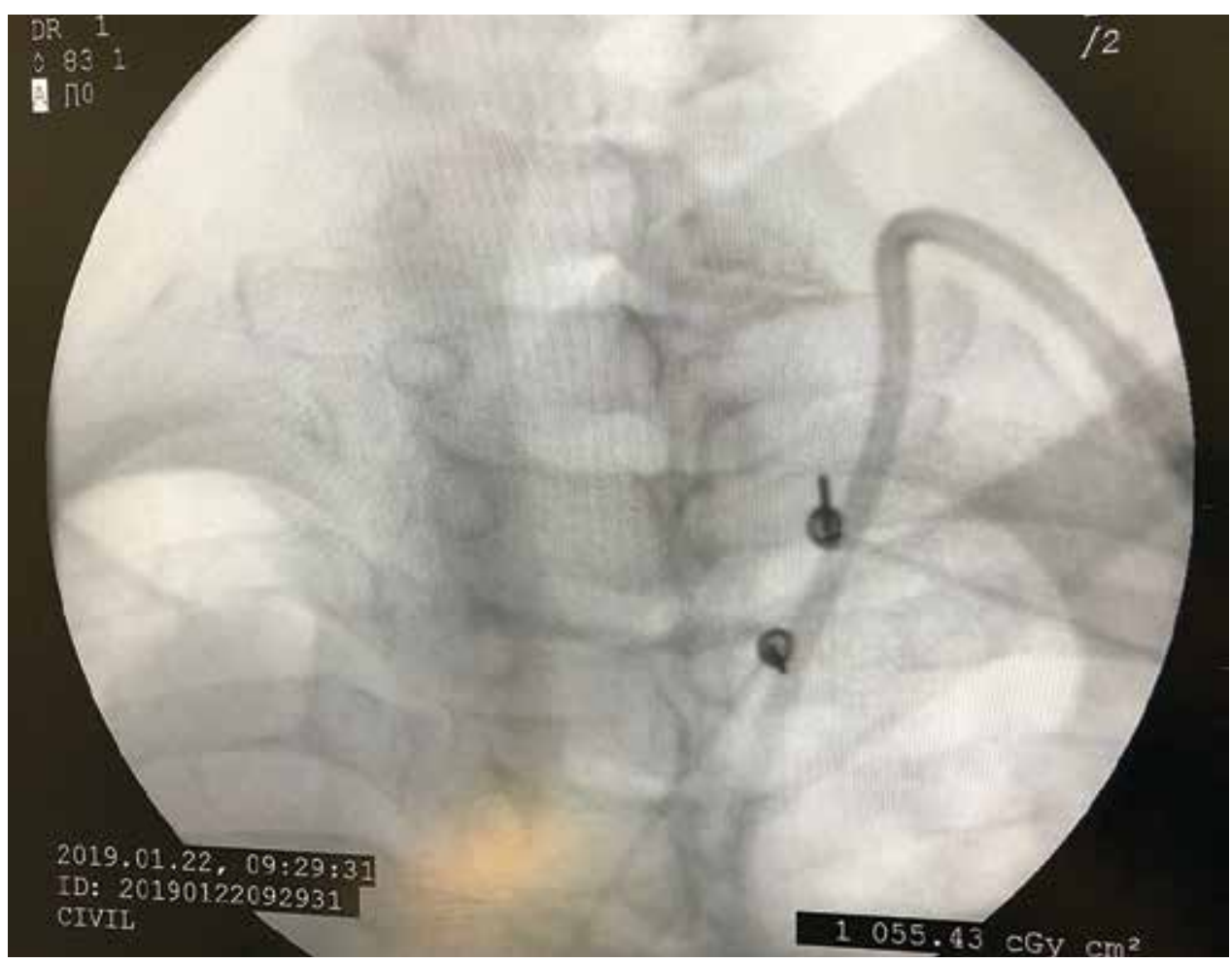

Fig. 3. Radiofrequency ablation for T2 compression fracture.

bilateral medial branch radiofrequency ablation of the $\mathrm{T} 1$ and T2 levels. After a positive test block with lidocaine, we performed medial branch ablation at each level using sensorial positive stimulation below $0.5 \mathrm{~V}$ and motor stimulus with multifidus contracture below $1.5 \mathrm{mV}$ (Fig. 3). Consequently, radiofrequency ablation of the T1 and T2 medial branches was performed with a bilateral lesion lasting 90 seconds at $80^{\circ} \mathrm{C}$.

A day after the intervention, we interviewed the patient, who experienced complete pain relief (VAS 0 ). The day after that, she was discharged from the hospital due to improvement in her respiratory and digestive symptoms and adequate pain control.

Three weeks later, the patient was newly admitted to the hospital because of a persistent cough and general respiratory deterioration. CT scan revealed new-onset pneumonia; hence, antibiotic treatment was completed with good results. Subsequently, the patient began to experience dorsal pain but at a lower location than before (VAS 8-10). MRI also showed a T6 compression fracture (Fig. 2). Vertebroplasty was again discarded/rejected by the Interventionist Radiology department because of poor visualization of anatomical structures. Further, the patient didn't respond to a progressive increase in opioid doses, so she was referred back to the Pain Unit. Radiofrequency ablation of the T5 and T6 medial branches was performed (Fig. 4) resulting in alleviation of pain at the dorsal area (VAS 2). One week later, her respiratory symptoms had improved with a larger than $50 \%$ reduction in opioid dosage (MED $100 \mathrm{mg}$ per day); thus, the patient was discharged from the hospital.

\section{RESULTS}

The patient left the hospital with managed pain (VAS 2). She was interviewed one month later and reported consistent pain alleviation (VAS 3) and lower opioid intake. In addition, she reported improved mood. Three months later, she reported continuing pain 


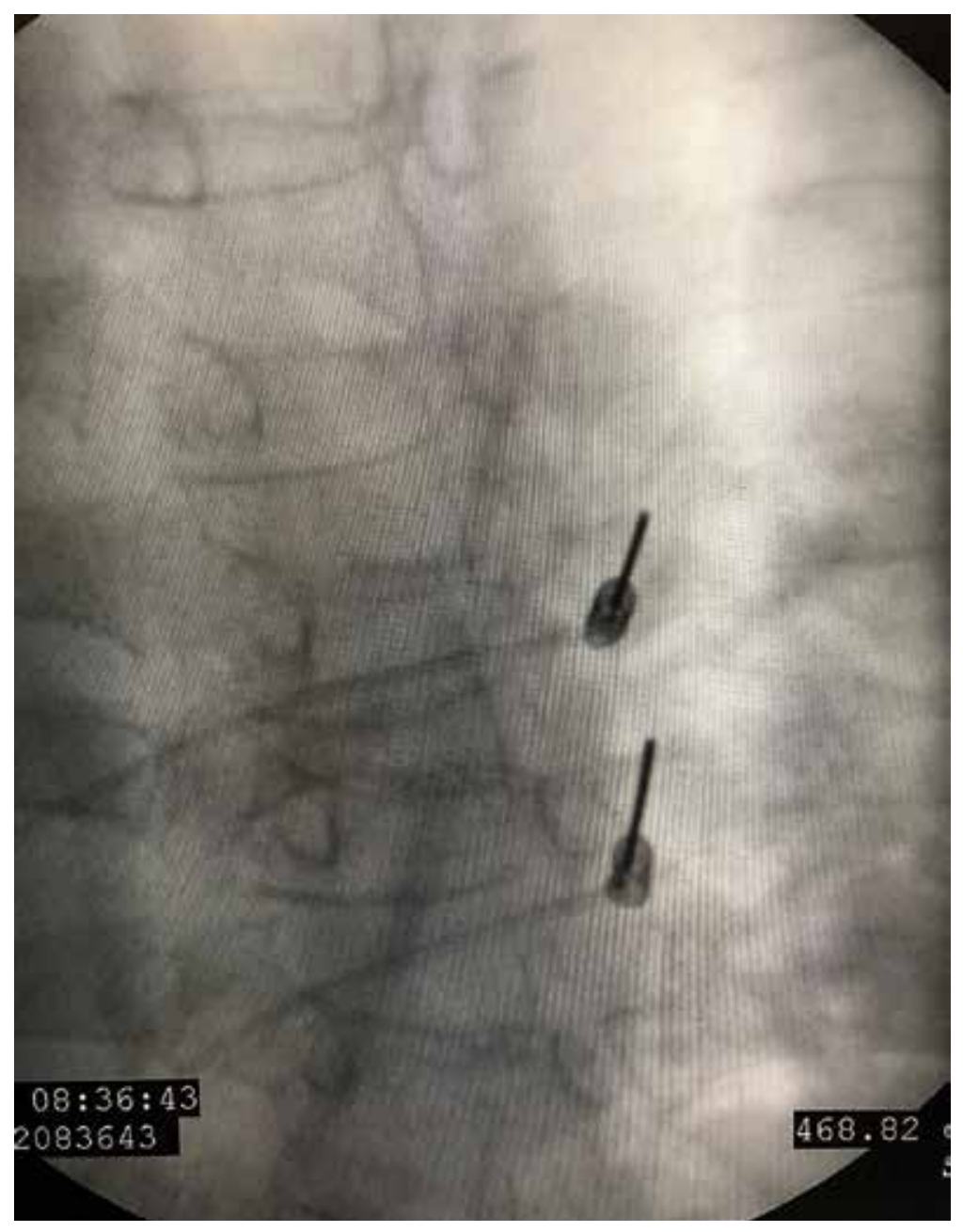

Fig. 4. Radiofrequency ablation for T6 compression fracture.

relief in the dorsal area, moderate lower back pain (VAS 4), and an improvement in her mood. Therefore, treatment was maintained with a MED of $75 \mathrm{mg}$ per day, clonazepam, prednisone, and denosumab, as well as bracing. Unfortunately, the patient died 2 weeks later due to adult respiratory distress syndrome (ARDS) secondary to a new pneumonia with controlled pain and a MED opioid dose of $50 \mathrm{mg}$ per day.

\section{DISCUSSION}

Osteoporotic vertebral compression fractures (VCF) are a common cause of mortality and morbidity in this segment of the population, occurring mostly in aging people. This implies a significant economic cost for the health care system and an increase in complications resulting from chronic pain and immobilization (5).

Treatment is conservative focusing on resting, analgesics, and bracing $(2,6)$. If pain persists, interventionist treatment should be initiated (7), consisting mainly of vertebral augmentation techniques: vertebroplasty and kyphoplasty $(8,9)$.

Percutaneous vertebroplasty (PVP) is a technique used to treat VCF that consists of introducing cement on a cannula, which is inserted through the pedicle. Lapras and Duquesnel established the first guidance indicators for this technique. Subsequently, kyphoplasty (a variation of vertebroplasty) was introduced. The method is similar, but involves the previous insertion of a balloon in the vertebral body, which, when inflated, can recover its height partially or even completely (9).

The effectiveness of these procedures in the treatment of pain secondary to VCF is high, resulting in partial pain relief in $87 \%$ of patients and short- and long-term improvement of physical function (10). The mechanism by which PVP produces analgesia is unknown. Several investigations have hypothesized about 2 alternatives: mechanical stabilization avoiding micromotion at the fracture site, and thermal necrosis or chemotoxicity of intraosseous pain receptors (11). Nevertheless, these techniques are still controversial and can lead to complications such us venous embolism by the cement, neurologic injury by cement leakage, and fractures of adjacent vertebral bodies (3). Moreover, in some cases, pain persists after vertebroplasty. This can have a multifactorial origin, one of the causes being anterior 
(disc) and posterior (facet joints) anatomical structures (12). Consequently, alternative techniques have been investigated to treat pain secondary to VCF.

Kim et al reported that facet joint block and posterior radiofrequency ablation prior to vertebroplasty could be a useful treatment for VCF. A high percentage of these patients showed prolonged pain relief (13). On the other hand, Park et al described the facet joint block to be beneficial in persistent pain treatment after PVP (14). Wang et al conducted the only retrospective study comparing the results between PVP and facet joint block. They observed that PVP obtained better results in short-term pain relief. Nevertheless, both techniques appeared to be equally effective in the long term (between a month and a year), as their results came to be similar. In addition, the facet joint block had a lower cost and fewer complications; thus, it was considered a useful treatment for severe pain secondary to VCF (15).

Furthermore, there have been changes described in the MRI signal in relation to acute and subacute facet joint fractures. This could support the theory that VCF builds up tension in the facet joints due to anatomical alterations produced mainly in the posterior elements (16). It appears that posterior vertebral elements and, especially, facet joints, could play an important role in developing pain secondary to VCF.

In fact, Bogduk et al developed a biomechanical model that explains the origin of pain secondary to VCF based on the role of the posterior elements. They believe the anatomical modification of said vertebral elements to be the source of pain. Thus, VCF alters the anterior column, which produces a cephalic or caudal subluxation of the posterior elements, causing pain (12). They affirm that anterior wedge fractures would lead to the damage of the upper facet joint due to anterior subluxation of its inferior process, which could strain the facet joint's capsule or even disrupt it (12).

In the case of VCF, there is not an anterior angulation but a loss of height in the anterior column, which results in the alteration of posterior elements of the lower facet joint. This occurs because, due to said loss of height, the inferior articular processes of the fractured vertebra and the superior articular process- es of the vertebra below come closer in such a way that the lamina of the fractured vertebra is level with the apex of the superior articular processes below. Pain can occur because of strain or disruption of the facet joints or periosteal irritation (12).

This model would explain pain secondary to VCF as a consequence of the damage to the posterior elements and not the vertebral body. This could be demonstrated by performing selective blocks with local anesthesia to medial branches of the dorsal branch - which innervate facet joints - of the fractured vertebra. It could even have prognostic value if we were to perform radiofrequency on said medial branches $(12,17)$.

In the case of our patient, given the predominantly vertical nature of the fractures and following Bogduk's model, we decided to focus on the lower joint. We performed T1 and T2 bilateral medial branch blocks, and in a second intervention we performed medial branch blocks with $0.5 \mathrm{~mL}$ of lidocaine $1 \%$ at T5 and T6. Both times we obtained almost complete and immediate pain relief. Subsequently, we performed bilateral radiofrequency ablation in said levels resulting in prolonged pain relief.

In addition, L3 vertebroplasty had previously been used to treat lumbar pain resulting in partial pain relief. However, this treatment could not be done again because of technical difficulties. Therefore, we performed $\mathrm{T} 2$ and $\mathrm{T} 6$ radiofrequency ablation, which resulted in prolonged pain relief.

Pain secondary to vertebral fracture might originate from the disruption of anterior as well as posterior vertebral elements, depending on the nature of the fracture. In the case of our patient, L3 vertebroplasty produced only partial pain relief, while T2 and T6 radiofrequency resulted in almost complete pain relief. This is why we propose infiltration and radiofrequency ablation of facet joints as an alternative approach in several circumstances: in acute as well as chronic VCF, which don't respond to conservative treatment; in cases that present technical difficulties or in which vertebral augmentation techniques are contraindicated; and lastly, as a complementary treatment for persistent pain subsequent to vertebroplasty (as an alternative to high doses of opioids or surgery). 
The use of radiofrequency ablation of facet joints has proved to be safer and free of complications (mostly neurological) as well as cheaper compared to vertebral augmentation techniques. Radiofreqency ablation would allow the use of these analgesic procedures in a higher percentage of patients with acute or chronic pain secondary to VCF.

\section{CONCLUSIONS}

Radiofrequency ablation of the medial branch after VCF can be an alternative to vertebral augmentation techniques in patients with technical problems, contraindication, or with persistent pain. In addition, it could be safer and more cost-effective compared to PVP.

\section{REFERENCES}

1. Johnell O, Kanis JA. An estimate of the worldwide prevalence and disability associated with osteoporotic fractures. Osteoporos Int 2006; 17:1726-1733.

2. Rzewuska M, Ferreira M, McLachlan AJ, Machado GC, Maher CG. The efficacy of conservative treatment of osteoporotic compression fractures on acute pain relief: A systematic review with meta-analysis. Eur Spine J 2015; 24:702-714.

3. Goldstein CL, Chutkan NB, Choma TJ, Orr RD. Management of the elderly with vertebral compression fractures. Neurosurgery 2015; 77:S33-S45.

4. Bodjuk N, MacVicar J, Borowczyk J. The pain of vertebral compression fractures can arise in the posterior elements. Pain Med 2010; 11:1666-1673.

5. Cummings SR, Melton LJ. Epidemiology and outcomes of osteoporotic fractures. Lancet 2002; 359:1761-1767.

6. Evans AJ, Jensen ME, Kip KE, et al. Vertebral compression fractures: Pain reduction and improvement in functional mobility after percutaneous polymethylmethacrylate vertebroplastyRetrospective report of 245 cases. Radiology 2003; 226:366372.

7. Klazen CA, Lohle PN, de Vries J, et al. Vertebroplasty versus conservative treatment in acute osteoporotic vertebral compression fractures (Vertos II): An open-label randomized trial. Lancet 2010; 376:1085-1092.

8. Lapras C, Mottolese C, Deruty R, Lapras C Jr, Remond J, Duquesnel J. Percutaneous injection of methyl-metacrylate in osteoporosis and severe vertebral osteolysis (Galibert's technic). Ann Chir 1989; 43:371-376.

9. Garfin SR, Yuan HA, Reiley MA. New technologies in spine: Ky- phoplasty and vertebroplasty for the treatment of painful osteoporotic compression fractures. Spine 2001; 26:1511-1515.

10. Hulme PA, Krebs J, Fergusson SJ, Berlemann U. Vertebroplasty and kyphoplasty: A systematic review of 69 clinical studies. Spine 2006; 31:1983-2001.

11. Georgy BA. Interventional techniques in managing persistent pain after vertebral augmentation procedures: A retrospective evaluation. Pain Physician 2007; 10:673-676.

12. Bogduk N, MacVicar J, Borowczyk J. The pain of vertebral compression fractures can arise in the posterior elements. Pain Med 2010; 11:1666-1673.

13. Kim TK, Kim KH, Kim CH, et al. Percutaneous vertebroplasty and facet joint block. J Korean Med Sci 2005; 20:1023-1028.

14. Park KD, Jee $\mathrm{H}$, Nam HS, et al. Effect of medial branch block in chronic facet joint pain for osteoporotic compression fracture: One year retrospective study. Ann Rehabil Med 2013; 37:191201.

15. Wang B, Guo H, Yuan L, Huang D, Zhang H, Hao D. A prospective randomized controlled study comparing the pain relief in patients with osteoporotic vertebral compression fractures with the use of vertebroplasty or facet blocking. Eur Spine ] 2016; 25:3486-3494.

16. Lehman V, Wood C, Hunt C, et al. Facet joint signal change on MRI at levels of acute/subacute lumbar compression fractures. Am J Neuroradiol 2013; 34:1468-1473.

17. Lau P, Mercer S, Govind J, Bodjuk N. The surgical anatomy of lumbar medial branch neurotomy (facet denervation). Pain Med 2004; 5:289-298. 